\title{
Análise crítica do desempenho evolutivo da visão subnormal no Instituto Benjamin Constant
}

\author{
Critical analysis of the progressive performance of low vision in \\ Benjamin Constant Institute
}

Helder Alves da Costa Filho ${ }^{1}$

Adriana Berezovsky ${ }^{2}$
Trabalho realizado no Instituto Benjamin Constant como pré-requisito à Tese do Mestrado Profissionalizante/MBA da Prática Oftalmológica da Universidade de São Paulo (UNIFESP). São Paulo (SP).

Médico Oftalmologista, responsável pelo Setor de Visão Subnormal do Instituto Benjamin Constant. Rio de Janeiro (RJ); Master in Business Administration de Gestão em Saúde e Mestre Profissional em Administração da Prática Oftalmológica da Universidade Federal de São Paulo (UNIFESP). São Paulo (SP).

${ }^{2}$ Professora Adjunta do Departamento de Oftalmologia e Doutora em Ciências pela UNIFESP. São Paulo (SP).

Endereço para correspondência: Helder Alves da Costa Filho - Praia do Flamengo, 300 - Apto. 1001 - Rio de Janeiro (RJ) CEP 22.210-030

E-mail: heldercostafilho@ig.com.br

Recebido para publicação em 27.04.2004

Versão revisada recebida em 11.05.2005

Aprovação em 23.09.2005

Nota Editorial: Depois de concluída a análise do artigo sob sigilo editorial e com a anuência da Dra. Keila Miriam Monteiro de Carvalho sobre a divulgação de seu nome como revisora, agradecemos sua participação neste processo.

\section{RESUMO}

Objetivo: Avaliar a efetividade do setor de Visão Subnormal no Instituto Benjamin Constant (IBC) e comprovar a real necessidade de um Instituto nos moldes do Instituto Benjamin Constant na atual política de inclusão. Métodos: Estudo ecológico, realizado de 1ํ de outubro de 1990 a 20 de dezembro de 2002, analisando três períodos de atendimento no setor de Visão Subnormal no Instituto Benjamin Constant: a) 1991 - início do atendimento; b) 1995 - integração médico-pedagógica; c) 2002 - estágio atual. Nesta análise foram estudados os seguintes indicadores: I - número de atendimentos no setor de Visão Subnormal; II - condições do setor de Visão Subnormal no Instituto Benjamin Constant e III - associados. Resultados: Observou-se aumento do atendimento, que passou a ser mais abrangente após a integração médico-pedagógica. Outros indicadores, como a capacitação de profissionais médicos, a participação em cursos de capacitação no Instituto Benjamin Constant, o aumento do número de convênios e encaminhamentos para o Instituto Benjamin Constant, além da Reabilitação, também confirmam a efetividade do setor de Visão Subnormal no Instituto Benjamin Constant. Conclusões: O setor de Visão Subnormal mostrou ser a interface entre o serviço médico e o serviço pedagógico, passando a interagir posteriormente com a Reabilitação e a Coordenação de Educação Física. Isto acarretou mudança de postura de diferentes setores do Instituto Benjamin Constant com relação ao paciente portador de visão subnormal, desde o educando até o paciente da comunidade. Desse modo, o Instituto Benjamin Constant mostrou a sua utilidade no tocante à política de inclusão.

Descritores: Baixa visão; Cegueira; Portadores de deficiência visual/reabilitaçao; Serviços de informação; Academias e Institutos; Efetividade

\section{INTRODUÇ̃̃OO}

O histórico da cegueira mostra desde o abandono de filhos (prática de "exposição") ${ }^{(1-2)}$ a honrarias de videntes e profetas ${ }^{(3-5)}$. O atendimento a essas pessoas se iniciou de forma caritativa ao longo da Idade Média, sob proteção da Igreja, sendo gradativamente assumido pelas autoridades civis, surgindo assim as primeiras instituições asilares para dar assistência e proteção às pessoas deficientes ${ }^{(1,3,6)}$. Este atendimento evoluiu com a criação de escolas para cegos a partir de 1784 em Paris, com o nome de Instituto Real dos Jovens Cegos e no século seguinte como as de Edimburgo e Berlim ${ }^{(1,3,6-7)}$.

A primeira Escola de Cegos na América do Sul, foi criada no Rio de Janeiro em 1854 com o nome de Instituto Real dos Jovens Cegos, sendo hoje conhecida pelo nome de Instituto Benjamin Constant (IBC) ${ }^{(1,3,6,8-9)}$ que até hoje é considerado como centro de referência nacional na área de deficiência visual $^{(10)}$. Além de ter criado a primeira Imprensa Braille do Brasil (1926), o IBC 
serviu de modelo educacional para as primeiras escolas especiais para alunos $\operatorname{cegos}^{(1,3,6,8-9)}$. O fato de ser uma escola para cegos, proporcionou que fossem encaminhadas à Instituição, alunos portadores de deficiência que não eram cegos, o que trazia aos professores algum desconforto com relação ao sistema de ensino a empregar ${ }^{(7)}$.

A partir da década de 50 foram criadas no IBC, as Classes de Conservação da Visão $(\mathrm{CCV})$ de $1^{\underline{a}}$ a $4^{\underline{a}}$ série, nas quais os alunos que não eram cegos tinham professores videntes, usavam salas com quadro-negro, boa iluminação e cadernos com pauta mais larga. Aos alunos do CCV era sugerido o aprendizado de Braille. A partir do ginásio, as turmas se tornavam mistas (CCV e cegos), com pouca utilização do quadro-negro.

Preocupado com a magnitude do problema de educação das pessoas portadoras de deficiência visual e das dimensões continentais do Brasil, o IBC passou a se preocupar com a especialização de professores; alguns de outras regiões do país para que retornassem aos lugares de origem irradiando conhecimentos, difundindo técnicas e transmitindo experiências adquiridas em cursos e estágios ali realizados ${ }^{(8)}$.

O IBC atualmente é um órgão específico singular da Administração Federal Direta, subordinado ao Ministro de Estado de Educação com supervisão e todo o orçamento de responsabilidade do MEC. Em algumas atividades pontuais o IBC conta com parcerias; em 2002 foram realizadas parcerias com o Lions Internacional e a Hellen Keller Internacional ${ }^{(10)}$.

Além da escola o IBC apresenta objetivos redirecionados e dimensionados. Produz e difunde o conhecimento na área, capacitando profissionais, assessorando escolas e instituições, realizando atendimento clínico, cirúrgico e de reabilitação e produzindo material especializado em Braille e publicações científicas.

A partir de 1990 foi iniciado no Serviço Médico do IBC o atendimento de visão subnormal (VSN), que segundo a Organização Mundial de Saúde, atinge 135 milhões dos 180 milhões de deficientes visuais do mundo.

De acordo com o IBGE 2000 um número maior de portadores de deficiência do que o esperado: 24,5 milhões de pessoas (cerca de $14,5 \%$ ) da população brasileira ${ }^{(11)}$. O conceito utilizado de deficiência na pesquisa seguiu recomendações da Organização Mundial de Saúde (OMS) e da Organizações das Nações Unidas (ONU), que considerou os portadores de alguma deficiência mais branda e também os casos mais graves de incapacidade total (cegueira, surdez, etc.).

Dentre as deficiências, a visual apresentou uma participação maior com 48,1\% (16.573.937), a motora 7.879.601, a auditiva 5.750.809, a mental 2.848.684 e a física 1.422 .224 .

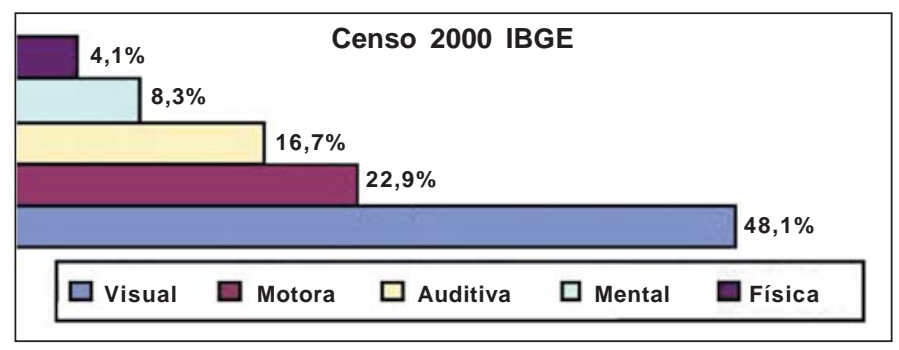

Face a estes dados e a Política de Inclusão ${ }^{(12)}$, este estudo tem como objetivos:

1. Avaliar a efetividade do atendimento de visão subnormal no IBC no período de 1990 até 2002;

2. Verificar o impacto da visão subnormal no Instituto Benjamin Constant;

3. Avaliar a real necessidade de um instituto nos moldes do IBC na atual Política de Inclusão.

\section{MÉTODOS}

O Estudo Ecológico Exploratório de tendência temporal foi a metodologia aplicada para avaliar a efetividade do atendimento de visão subnormal no Instituto Benjamin Constant no período de $1^{\circ}$ de outubro de 1990 a 20 de dezembro de 2002 . $^{(13)}$ Serão analisados três períodos:

1. 1991 - Início do atendimento (01/10/1990 a 16/12/1991) Início do atendimento em uma sala do serviço médico dividida com outras especialidades.

2. 1995 - Integração médico-pedagógica (10/01/1995 a 22/11/ 1995)

Apesar do atendimento em conjunto com professores e da aquisição de alguns recursos ópticos terem ocorrido ao longo de 1994, foi em 1995 que a integração médico-pedagógica estava consolidada.

3. 2002 - Estado atual (06/03/2002 a 20/12/2002)

A proposição de avaliar o estado atual para dar base a um estudo analítico, comparativo com relação aos outros períodos.

Nestes três períodos, foram considerados como indicadores:

\section{1 - Atendimentos na Visão Subnormal no IBC}

Atendimentos realizados pelo médico especialista em visão subnormal. Todos os pacientes foram atendidos e tratados no Serviço Médico anteriormente.

1.1 Atendimento total

1.1.1 Número de atendimentos - total de atendimentos na Visão Subnormal, ao longo do ano (novos e retornos)

1.2 Atendimentos novos

1.2.1 Número de atendimentos novos - pacientes atendidos pela primeira vez ao longo do ano

1.2.2 Origem dos pacientes novos:

1.2.2.1 Pacientes de Estimulação Visual - Crianças na faixa etária de 0 a 5 anos, atendidas na Estimulação Precoce do IBC.

1.2.2.2 Alunos do Instituto - alunos matriculados regularmente no IBC no ano do primeiro atendimento

1.2.2.3 Alunos de escola regular - alunos portadores de deficiência visual regularmente matriculados em escola regular quando do primeiro atendimento na Visão Subnormal

1.2.2.4 Comunidade - pacientes da comunidade que eram atendidos no Serviço Médico do IBC.

1.2.2.5 Reabilitandos do Instituto - pacientes regularmente matriculados na Reabilitação do IBC quando do primeiro atendimento na VSN. Alguns são ex-alunos do IBC. 
1.2.3 Grupo etário dos pacientes novos. Foram considerados: 0 a 5 anos, 6 a 10 anos, 11 a 20 anos, 21 a 30 anos, 31 a 40 anos, 41 a 50 anos, 51 a 60 anos e maior do que 60 anos.

1.2.4 Pacientes da comunidade em idade escolar (4 a 20 anos) que não estavam freqüentando escola na ocasião do primeiro atendimento ${ }^{(14)}$.

\section{2 - Condições na Visão Subnormal no IBC}

Foram considerados, entre outros a subordinação da VSN, o espaço físico, as condições de atendimento, os recursos ópticos, a integração com a Pedagogia, a participação de residentes, estagiários e outros.

\section{3 - Associados}

Foram abordados os seguintes aspectos:

3.1 Capacitação

3.1.1 Capacitação de profissionais - estágio de profissionais no atendimento (oftalmologistas, ortoptistas e psicólogos)

3.1.2 Participação em cursos de capacitação e treinamento de recursos humanos do Instituto Benjamin Constant - participação da equipe de visão subnormal em cursos de capacitação de recursos humanos do IBC

3.2 Convênios e encaminhamentos para atendimento no IBC - foram considerados os pacientes encaminhados por convênios com o IBC e os que foram simplesmente encaminhados por outros Institutos, Escolas Especiais ou não, Secretarias de Educação e Prefeituras.

3.3 Evolução da Reabilitação - A Reabilitação do IBC foi criada em $1994^{(15)}$.

Considerou-se o número de pacientes atendidos ao longo do ano, bem como o número de atendimentos oferecidos (Orientação e Mobilidade, Sistema Braille e Afinação de Piano, dentre outros) e o número de cursos disponíveis (Massoterapia, Telefonista, Empalhação, dentre outros).

\section{RESULTADOS}

Os resultados dos indicadores serão mostrados por três períodos:

1991 - Início do atendimento

1995 - Integração médico-pedagógica

2002 - Estado atual

\section{1 - Atendimentos}

\begin{tabular}{|lcrc|}
\hline \multicolumn{4}{|c|}{ Indicadores nos três períodos } \\
& $\mathbf{1 9 9 1}$ & $\mathbf{1 9 9 5}$ & $\mathbf{2 0 0 2}$ \\
Número de atendimentos & 86 & 385 & 473 \\
Número de atendimentos novos & 51 & 157 & 206 \\
Pacientes da Estimulação Visual & 0 & 6 & 186 \\
Alunos do IBC & 36 & 17 & 28 \\
Alunos da Escola Regular & 0 & 46 & 57 \\
Comunidade & 15 & 76 & 92 \\
Reabilitandos do IBC & 0 & 12 & 11 \\
Pacientes da comunidade (4 a 20 anos) & 0 & 26 & 9 \\
que não estavam freqüentando a escola & & \\
\hline
\end{tabular}

O gráfico a seguir mostra o grupo etário dos pacientes novos nos três períodos estudados.



\section{2 - Condições na Visão Subnormal}

\section{1}

1. A Visão Subnormal era ligada ao Serviço Médico de Oftalmologia do IBC, que por sua vez era subordinado ao Departamento de Atendimento ao Educando (DAE).

2. $\mathrm{O}$ atendimento era realizado em uma sala ampla do Ambulatório de Oftalmologia que era dividida com outros colegas que faziam a anamnese dos seus pacientes.

3. Não havia integração com a Pedagogia e eram poucos os recursos ópticos disponíveis para o exame.

4. O Serviço Médico forneceu uma armação de provas e uma caixa de lentes. Havia, até então, poucos recursos ópticos e as tabelas não eram específicas para VSN.

5. A pedido dos residentes do IBC, o atendimento que era só para alunos do IBC, se estendeu para pacientes da comunidade que eram atendidos no Serviço.

\section{5}

1. A Visão Subnormal fazia parte do Departamento Médico, Nutricional e de Reabilitação através da Divisão de Atendimento Médico Oftalmológico e de Reabilitação.

2. A integração médico-pedagógica era feita pela Coordenação de Estimulação da Visão Funcional de Educandos e Reabilitandos em conjunto com a Coordenação de Atendimento Oftalmológico.

3. O Setor dispunha de duas salas exclusivas, adaptadas com recursos ópticos adquiridos no ano anterior.

4. Participação na matrícula do IBC de candidatos a aluno.

5. Atendimento em três períodos em conjunto com cinco professoras do IBC.

6. Participação dos residentes do $2^{\circ}$ ano.

7. Treinamento de recursos ópticos pelos professores.

8. Professores faziam o elo nos diferentes segmentos:

Estimulação Precoce - 0 a 4 anos

Jardim de Infância - 4 a 7 anos

Classe de Alfabetização - 8 a 12 anos

Ensino Fundamental ( $1^{\underline{a}}$ fase $)-1^{\underline{a}}$ a $4^{\underline{a}}$ série

Ensino Fundamental ( $2^{\underline{a}}$ fase $)-5^{\underline{a}}$ a $8^{\underline{a}}$ série

Múltipla Deficiência 
9. Utilização dos recursos ópticos em sala de aula pelos alunos do IBC.

10. Utilização dos CCTVs pelos alunos e reabilitandos do IBC.

11. Estudo e capacitação da equipe de visão subnormal para utilização do teste de Teller.

12. Classificação visual de atletas encaminhados pelo Departamento de Educação Física.

\section{2}

1. A Visão Subnormal era subordinada ao Departamento de Estudos e Pesquisas Médicas e de Reabilitação (DMR) na Divisão de Reabilitação, Preparação para o Trabalho e Encaminhamento Profissional (DRT).

2. O Setor dispunha de uma sala exclusiva para o atendimento, enquanto a outra era dividida com as especialidades de Lente de contato e Plástica ocular, Vias lacrimais e Órbita.

3. Não foram adquiridos recursos ópticos desde 1995. Os novos recursos foram doados pela equipe de visão subnormal.

4. Não havia participação na matrícula e sim na avaliação dos alunos já matriculados e candidatos a aluno para o ano seguinte.

5. Atendimento em dois períodos com duas professoras que passaram a fazer a ligação com todos os segmentos escolares.

6. Participação dos residentes do $2^{\circ}$ ano.

7. Redução das sessões de treinamento de recursos ópticos

8. Utilização dos recursos ópticos em sala de aula pelos alunos do IBC.

9. Utilização de dois CCTVs pelos alunos e reabilitandos do IBC.

10. Classificação visual de atletas encaminhados pela Coordenação de Educação Física do IBC.

\section{3 - Associados}

\subsection{Capacitação}

3.1.1 Capacitação de profissionais

1991 - Não havia

1995 - Estágio de uma psicóloga

2002 - Estágio de oftalmologistas com título de especialista

3.1.2 Participação em cursos de capacitação e treinamento de recursos humanos do Instituto Benjamin Constant

1991 - Não havia participação nos cinco cursos

1995 - Participação em dois dos nove cursos (um no município de Juiz de Fora/MG)

2002 - Participação em sete dos 15 cursos (três no município de Angra dos Reis/RJ)

3.2 Convênios e encaminhamentos para atendimento no IBC

1995 - Instituto Nacional de Educação de Surdos

- Sodalício de Sacra Família/RJ

- 17 municípios do Estado do Rio de Janeiro (exceto a capital)
2002 - Instituto Helena Antipoff/RJ

- Instituto Nacional dos Educação de Surdos

- 26 municípios do Estado do Rio de Janeiro (exceto a capital):

- Minas Gerais - 1

- Acre - 1

3.3 Evolução de Reabilitação

1991 - Não havia sido criada

1995 - 131 pessoas atendidas:

1. nove tipos de atendimentos

2. nove tipos de cursos

2002 - 194 pessoas atendidas:

1. 13 tipos de atendimentos

2. quatro tipos de cursos

\section{DISCUSSÃO}

Este estudo propõe uma análise crítica do desempenho evolutivo da Visão Subnormal no IBC, desde o início do atendimento até o ano de 2002, buscando avaliar a efetividade, os fatores externos e o papel da Visão Subnormal dentro da Política Nacional de Inclusão.

\section{1 - Atendimentos}

Foi observado um aumento progressivo no número de atendimentos nos 12 anos da VSN no IBC, apesar da diminuição do número de períodos de atendimento entre 1995 e 2002.

$\mathrm{O}$ aumento observado nos pacientes encaminhados pela Estimulação Visual está diretamente relacionado à estruturação do setor com recursos, material humano e ao encaminhamento cada vez mais precoce para avaliação oftalmológica e cuidados visuais.

A diminuição no número de atendimentos de alunos novos do IBC em 1995, se deve ao fato da VSN ter atuado na matrícula. Cabe ressaltar que alguns alunos que foram para a escola regular e outros que não necessitaram ser matriculados no IBC continuaram a ser acompanhados pela VSN.

O fato de não haver a participação da VSN na matrícula e no atendimento dos alunos novos explica porque no Relatório de atividades da Visão Subnormal de julho de 1994(16), foi feito menção a quatro alunos que estavam sendo educados no Sistema Braille e que poderiam estar no Sistema comum de Leitura e Escrita.

$\mathrm{O}$ aumento no atendimento de alunos de escola regular e de pacientes da comunidade se relaciona ao conhecimento pelas Instituições de Ensino, dos professores, residentes do IBC, oftalmologistas de outros serviços, médicos de outras especialidades, pacientes e de seus familiares quanto à possibilidade de melhoria da visão funcional com os recursos da VSN.

Ainda é observado com freqüência o desconhecimento dos pacientes com relação à causa da deficiência visual e limitações de resultados na VSN, o que gera uma grande expectativa. Isto também ocorre com relação aos oftalmologistas com relação às indicações para o tratamento. 
Observou-se a estabilização no atendimento aos reabilitandos de 1995 para 2002 que estava relacionado ao alto índice de absenteísmo. Constatou-se na troca do dia de atendimento de $6^{\underline{a}}$ feira para $4^{\underline{a}}$ feira no ano de 2003 , que era pela dificuldade de vir ao IBC em um dia fora das atividades normais da Reabilitação no IBC.

Com relação ao grupo etário dos pacientes novos, ressaltaríamos que o aumento no número de pacientes acima de 60 anos, também está relacionado à motivação e necessidade de serem mais participativos em atividades de trabalho e lazer. A estabilização na faixa de 6 a 10 anos e a diminuição na de 11 a 20 anos está explicada pelo fato de terem sido as mais numerosas em 1995 e só estarmos contabilizando pacientes novos.

$\mathrm{O}$ aumento da procura e oferta de cursos para professores em função da Política de Inclusão e o melhor preparo dos mesmos explicaria a diminuição do número de pacientes da comunidade na faixa de 4 a 20 anos que não estavam freqüentando a escola quando do atendimento.

\section{2 - Condições na Visão Subnormal}

Concluímos que a integração médico-pedagógica foi um marco nesta evolução. A aproximação dos dois setores além de trazer um grande benefício para as duas partes, melhorou o atendimento.

\section{Setor Médico}

1. Conhecimento da importância de se trabalhar o resíduo visual;

2. Conhecimento das necessidades do professor em sala de aula;

3. Indicação dos pacientes para visão subnormal (Como? Quando?);

4. Disponibilidade de consultoria em visão subnormal;

5. Como orientar o paciente cego;

6. Importância da estimulação visual;

7. Importância da reabilitação. Normalmente a formação médica enfoca com maior ênfase a cura e a prevenção;

8. Conhecimento do uso e dos limites dos recursos ópticos.

\section{Setor Pedagógico}

9. Foi necessária uma reunião com o corpo docente do IBC no início da integração médico-pedagógica, para explicar o que era visão subnormal e acabar com o mal-entendido que fazia com que os alunos do IBC faltassem ao atendimento, pois foi divulgado que o objetivo seria retirá-los do Instituto;

10. Melhora no relacionamento com o Serviço Médico. Havia distanciamento que fazia com que os professores não se sentissem à vontade;

11. Conhecimento das patologias de base, do tratamento e da repercussão funcional na visão;

12. Conhecimento das vantagens e desvantagens dos recursos ópticos;

13. Disponibilidade para uso dos recursos ópticos dentro do Instituto;

14. Valorização da visão residual, mesmo que pequena, em atividades de vida independente e na educação, mesmo quando havia indicação de Braille;
15. Desmistificação pela maioria dos docentes de mitos como: "sentar próximo a TV faz mal", "não se pode usar o resíduo visual, pois se não economizá-lo a visão acaba mais rápido", "é mais fácil e econômico ensinar Braille para todos os portadores de deficiência do que usar recursos ópticos" etc. Houve um esvaziamento administrativo de Visão Subnormal no ano de 2002:

16. Diminuição do número de profissionais e do tempo por eles despendido;

17. Diminuição do espaço físico;

18. Não houve aquisição de recursos ópticos. Foram adquiridas duas tabelas em 2002;

19. Não houve manutenção dos recursos ópticos;

20. Mudança da coordenação de Estimulação à visão funcional de educandos e reabilitandos e ausência a partir de setembro.

Felizmente este esvaziamento não afetou a melhoria do atendimento, capacitação dos profissionais e outros indicadores. A compreensão do papel da Visão Subnormal mudou a postura dos profissionais do IBC que direta ou indiretamente estavam envolvidos.

A interação com outros setores, como a Coordenação de Educação Física, foi extremamente gratificante não só pelo trabalho de classificação oftalmológica para esportes, como pela orientação nas limitações de atividades físicas em função da patologia de base e pelo nosso aprendizado das dificuldades técnicas dos professores e técnicos da área. A partir daí, a Visão Subnormal passou a fazer parte dos cursos de capacitação de educação física e desporto.

\section{3 - Associados}

3.1 Capacitação

3.1.1 Capacitação de profissionais

Tivemos ao longo desses anos como estagiários uma mestranda em psicologia pela UERJ, ortoptistas, uma enfermeira de Belém do Pará e vários médicos oftalmologistas.

Alguns estagiários iniciaram o atendimento de VSN em outras clínicas, hospitais e consultórios. Dentre eles podemos citar o Hospital Universitário Clementino Fraga Filho da Universidade Federal do Rio de Janeiro, o Programa de Reabilitação e Estimulação pela Prefeitura do município de Juiz de Fora (MG), o Hospital Universitário Pedro Ernesto da Faculdade de Ciências Médicas da Universidade do Estado do Rio de Janeiro e atualmente o Hospital dos Servidores do Estado do Rio de Janeiro.

3.1.2 Participação em cursos de capacitação e treinamento de recursos humanos do Instituto Benjamin Constant.

A participação aumentou nos cursos ministrados dentro e fora do IBC pela credibilidade adquirida em setores como Pedagogia, Educação Física e Reabilitação e pela Política Nacional de Inclusão. 


\subsection{Convênios e encaminhamentos para atendimento}

Além do conhecimento das possibilidades da Visão Subnormal, a Lei de Diretrizes e Bases ${ }^{(14)}$ com a obrigatoriedade de atendimento educacional especializado gratuito aos educandos com necessidades especiais, preferencialmente na rede regular de ensino (Lei no ${ }^{\circ}$. 9.394/96) art. 4º, III) e o reconhecimento do IBC como centro de referência ${ }^{(10)}$ podem explicar o aumento.

\subsection{Reabilitação}

$\mathrm{O}$ aumento do número de atendimentos e de tipos de atendimentos atesta o reconhecimento da importância da Reabilitação pelo aumento da procura e nos possibilitou oferecer este atendimento a muitos pacientes que eram atendidos na VSN.

\section{CONCLUSÕES}

1. Este estudo mostrou a efetividade do Setor de Visão Subnormal do Instituto Benjamim Constant nos 12 anos estudados. Apesar do esvaziamento administrativo e da demanda crescente do serviço, a Visão Subnormal manteve um bom padrão de atendimento e desenvolvimento.

2. A Visão Subnormal revelou ser a interface entre o Serviço Médico e o Pedagógico e posteriormente com a Reabilitação e a Coordenação de Educação Física.

3. Houve uma maior conscientização e mudança clara de atitude nos Serviços Médico, Pedagógico e em outros setores do Instituto Benjamim Constant com relação à valorização do portador de visão subnormal.

4. Um Instituto nos moldes do Benjamin Constant justifica a sua existência, servindo de base para a Política de Inclusão do portador de deficiência visual nos níveis educacional, social, profissional e de capacitação de recursos humanos para profissionais da área.

\section{ABSTRACT}

Purpose: To evaluate effectiveness of the Low Vision the Benjamin Constant Institute (BCI) and confirm the real necessity of an Institute like BCI in the present inclusion policy. Methods: Ecological study, analyzing 3 periods of Low Vision Assistance at the Benjamin Constant Institute from October 1, 1990 to December 20, 2002: a) 1991 - starting assistance; b) 1995 - medical pedagogic integration; c) 2002 - present-day situation. We considered in this analysis as indicators: I Low Vision Assistance, II - Low Vision sector in the Benjamin Constant Institute, III - Associates. Results: This study demonstrated an increase in assistance, reaching a wider spectrum of patients after medical-pedagogic integration. Other indicators, such as physician capacitation, participation in Benjamin Constant Capacitation Courses, increase in orienta- tion to institutions, schools and others and referrals to the Benjamin Constant Institute, and Rehabilitation also attest the effectiveness of the Low Vision sector of the Benjamin Constant Institute. Conclusions: The Low Vision sector proved to be the interface between the Medical and Pedagogic Departments, and later on the Rehabilitation and Physical Education Coordination sectors. This has implied alterations in the way to manage the low-vision patient, not only regarding the regular Benjamin Constant Institute student as well as any other patient in the community. The Benjamin Constant Institute proved its importance as regards inclusion policy.

Key words: Vision, low; Blindness; Visually impaired persons/ rehabilitation; Information services; Academies and Institutes; Effectiveness

\section{REFERÊNCIAS}

1. Sombra LA. Evolução histórica das concepções sobre as pessoas excepcionais. Rio de Janeiro; 1982.

2. French RS. A social and educational study for the blind. Louisville, Kentucky: American Printing House of the Blind; 1932.

3. Bruno MMG, Moda MGB.; Instituto Benjamin Constant. Programa de capacitação de recursos humanos do ensino fundamental: deficiência visual. Brasília: Ministério da Educação, Secretaria de Educação Especial; 2001. vol. 1 n.1-3.

4. Veiga JE. Os fundamentos da instrução para cegos. In: Veiga JE. O que é ser cego: a situação dos cegos. Rio de Janeiro: J. Olympio; 1983. p.25-8.

5. Rocha H. Páginas esparsas IV de cegueira e cegos ilustres II. Belo Horizonte: Fundação Hilton Rocha; 1990. 278p.

6. Leite Filho LAM. O deficiente visual do Instituto Benjamin Constant e suas reações emocionais face a perda da visão, o tabagismo e a prótese ocular.: uma contribuição ao estudo da cegueira [tese]. Belo Horizonte: Faculdade de Medicina da Universidade Federal de Minas Gerais; 2000.

7. Scholl GT. A educação de crianças com distúrbios visuais. In: Educação de excepcionais: a educação da criança e do jovem excepcional. Rio de Janeiro: Globo; 1982.

8. Lemos FM, Ferreria PF. Instituto Benjamin Constant. Uma história centenária. Rev Benjamin Constant. 1995;(1):3-8.

9. Bruno MMG. Deficiência visual: reflexão sobre a prática pedagógica. São Paulo: Laramara; 1977.

10. Instituto Benjamin Constant [home page]. Rio de Janeiro: Instituto Benjamin Constant; 2003. [citado 2003 Dez 10]. Disponível em: http://www.ibcnet.org.br

11. Instituto Brasileiro de Geografia e Estatística. Censo 2000: incidência de deficiência visual. Brasília: IBGE; 2003. [citado 2003 Dez 10]. Disponível em: http://www.ibge.gov.br

12. Brasil. Ministério da Educação e Cultura. Brasília: MEC; 2003. [citado 2003 Dez 10]. Disponível em: http://www.mec.gov.br

13. Medronho RA de. Estudos ecológicos. In: Medronho RA de, organizador. Epidemiologia. São Paulo: Atheneu; 2002.

14. Brasil. Ministério da Educação. Secretaria da Educação Especial. CNE 02/01. Diretrizes nacionais para a educação especial na educação básica. Brasília: MEC; 2001. [citado 2003 Dez 10]. Disponível em: http://www.mec.gov.br/ seesp/Ftp/Diretrizes.pdf

15. Brasil. Ministério da Educação e do Desporto. Instituto Benjamin Constant. Portaria no. 398 de 15 de março 1994. Aprovar o regimento interno do Instituto Benjamin Constant. Diário Oficial da República Federativa do Brasil, Brasília, DF, n.51, 16 de março 1994, seção 4.

16. Strauss R. Projeto de visão subnormal [Relatório]. Rio de Janeiro: Instituto Benjamin Constant; 13 de julho de 1994. 